\title{
Experimental and Numerical Analysis of the Free Surface in a Water Model of a Slab Continuous Casting Mold
}

\author{
Raul MIRANDA, Miguel Angel BARRON, Jose BARRETO, ${ }^{1)}$ Luis HOYOS and Jesus GONZALEZ \\ Division de Ciencias Basicas e Ingenieria, Universidad Autonoma Metropolitana-Azcapotzalco, Apdo. Postal 16-218, C.P. \\ 02011, Mexico, D.F., Mexico. E-mail: gtji@correo.azc.uam.mx $\quad$ 1) Instituto Tecnologico de Morelia, Av. Santiaguito 1500 ,
} Morelia, Mexico.

(Received on May 20, 2005; accepted on July 29, 2005)

\begin{abstract}
The flow patterns prevailing at the free-surface in a water model of a slab continuous casting mold using several water flow rates and entry nozzle submergence depths are experimentally and numerically studied in this work. The experimental study was carried out using an one-third scale cold water model, constructed in accordance with the Froude similarity criterion. Water level measurements were carried out with ultrasonic distance sensors and recorded in a computer. Numerical simulations were made with a commercial computational fluid dynamics software. It was found that free-surface oscillations are composed by several periodic components. There exists a fundamental periodic frequency of $1.2 \mathrm{~Hz}$. Besides, there exist two other frequencies of 1.8 and $2.1 \mathrm{~Hz}$ whose contribution to the free surface dynamic behavior depend on the spatial position and on the process parameters, namely, the volumetric flow rate and the submerged entry nozzle (SEN) submergence depth. In accordance with the obtained results, several recommendations about operating policies of actual industrial casters are made.
\end{abstract}

KEY WORDS: free surface velocity; mold flow; power density spectrum; slab continuous casting; submerged entry nozzle.

\section{Introduction}

Given that steelmakers are aiming for higher casting speeds of liquid steel in continuous casting operations, it is of basic importance to establish the right operating conditions in order to obtain a clean and sound product. Therefore, catastrophic operations such as slag trapping, mold emptying and mold spilling over must be strictly avoided. In recent years the concept of smart mold has arisen. A key feature of the smart mold is that related with the full automation of the molten steel level. Automatic regulation of the mold level requires precise measurements of the actual steel level, and this is not an easy task given that the surface of the liquid steel is continuously varying due to turbulence and mold oscillation. Therefore, to develop control strategies which satisfy very tight specifications, it is necessary to characterize the dynamic behavior of the freesurface of the mold in continuous casting machines. A very complete characterization of the free-surface in continuously cast steel billets was reported by Kumar et al. ${ }^{1)}$ However, a characterization similar applied to steel slab continuous casting machines has not been reported.

Industrially, the mold level can be measured using different principles. $^{2-6)}$ Eddy-current sensors are preferred because they provide accurate measurements and are easy to put into practice. Floating sensors or radiometric sensors may also be used. However, the first ones are subject to fast wear because they are in contact with the molten metal. The second ones are sensitive to powder thickness variations and their use requires safety precautions.

Because of the nearly equal kinematic viscosities of liquid steel and water, flow in the steel caster mould region has been studied extensively using water models, which are easier to operate and visualize ${ }^{7-9)}$ than current continuouscasting machines. The surface fluctuations in water molds have been studied using capacitance pressure sensors, with images recorded with a video camera and even with rulers. $^{10)}$

The aim of this work is to study the flow patterns prevailing at the steel-slag interface as a function of the casting speed and the submerged entry nozzle (SEN) submergence depth. To do this, the water-air interface in a water model was tracked through numerical computational fluid dynamic (CFD) simulations, and physical experiments in an onethird water model were made in order to validate the numerical simulations. Several studies of this kind have been previously reported, ${ }^{4,7,9,11)}$ however, in some of them the analysis was focused on the free surface whereas the flow patterns in the upper mold section were neglected. In some works, the analysis was focused on the flow patterns in the upper mold section but their influence on the free surface was not studied.

\section{Mathematical Modeling}

The flow of an incompressible fluid is described by the 
well-known Navier-Stokes equations, which in vectorial form are expressed as follows ${ }^{8,10)}$ :

$$
\frac{\partial\left(\rho u_{i}\right)}{\partial t}+\frac{\partial\left(\rho u_{i} u_{j}\right)}{\partial x_{j}}=-\frac{\partial p}{\partial x_{i}}+\frac{\partial}{\partial x_{j}}\left\{\mu_{\mathrm{eff}}\left(\frac{\partial u_{i}}{\partial x_{j}}+\frac{\partial u_{j}}{\partial x_{i}}\right)\right\}
$$

where $\rho$ is the fluid density, $u_{i}$ is the $i$ component of the fluid velocity $u, t$ is time, $x_{j}$ is $j$ spatial coordinate, $p$ is pressure, and $\mu_{\text {eff }}$ is the effective fluid viscosity. To maintain the mass balance in the system, the continuity equation must be solved:

$$
\frac{\partial u_{j}}{\partial x_{j}}=0
$$

Given that the experiments are carried out isothermally, the energy equation is not considered. The $K-\varepsilon$ turbulence model was selected for mathematical modeling, which is described by the following expressions:

$$
\begin{aligned}
& \rho u_{j} \frac{\partial K}{\partial x_{j}}=\frac{\partial}{\partial x_{j}}\left(\frac{\mu_{\mathrm{t}}}{\sigma_{k}} \frac{\partial K}{\partial x_{j}}\right)+\mu_{\mathrm{t}} \frac{\partial u_{j}}{\partial x_{i}}\left(\frac{\partial u_{i}}{\partial x_{j}}+\frac{\partial u_{j}}{\partial x_{i}}\right)-\rho \varepsilon \\
& \rho u_{j} \frac{\partial \varepsilon}{\partial x_{j}}=\frac{\partial}{\partial x_{j}}\left(\frac{\mu_{\mathrm{t}}}{\sigma_{\varepsilon}} \frac{\partial \varepsilon}{\partial x_{j}}\right) \\
& +C_{1} \mu_{\mathrm{t}} \frac{\varepsilon}{K} \frac{\partial u_{j}}{\partial x_{i}}\left(\frac{\partial u_{i}}{\partial x_{j}}+\frac{\partial u_{j}}{\partial x_{i}}\right)-C_{2} \frac{\varepsilon}{K} \rho \varepsilon \ldots
\end{aligned}
$$

In the above equations $K$ is the turbulent kinetic energy, $\varepsilon$ is dissipation rate of $K . \sigma_{K}, \sigma_{\varepsilon}, C_{1}$ and $C_{2}$ are constants whose values are $1.0,1.3,1.44$ and 1.92 respectively. ${ }^{8)}$ The fluid viscosity must be corrected for turbulence in the Navier-Stoke equations employing an effective viscosity $\mu_{\text {eff }}=\mu_{1}+\mu_{\mathrm{t}}$, where $\mu_{1}$ is the laminar viscosity and $\mu_{\mathrm{t}}$ is the turbulent viscosity. The latter can be determined as follows:

$$
\mu_{\mathrm{t}}=\rho C_{\mu} \frac{\kappa^{2}}{\varepsilon}
$$

where $C_{\mu}=0.09$ is a constant. The boundary conditions in the mathematical model were those recommended by Thomas et $a l .{ }^{8)}$ and are as follows: Inlet turbulence parameters at the inlet were $K=0.044 \mathrm{~m}^{2} \mathrm{~s}^{-2}$ and $\varepsilon=1.00 \mathrm{~m}^{2} \mathrm{~s}^{-3}$. At the outlet, the boundary condition was fixed in such a way that the mass balance was satisfied.

A commercial CFD software was employed for numerical solving of the above mathematical model. A two-dimensional mesh with 32500 nodes was created with a time step of $0.001 \mathrm{~s}$. A personal computer with $1 \mathrm{~GB}$ of RAM memory and $3.0 \mathrm{GHz} \mathrm{CPU}$ was utilized for the numerical simulations. A run for $70 \mathrm{~s}$ of real time required a CPU time of approximately $5 \mathrm{~h}$. In order to track the free surface profile, two-phase flow was considered and the Volume-of-fluid (VOF) model $^{10)}$ was chosen for tracking of the air-water interface.
Table 1. Model Geometric Dimensions.

\begin{tabular}{|l|c|}
\hline Dimension & Value \\
\hline SEN bore diameter & $0.025 \mathrm{~m}$ \\
\hline Port diameter & $0.02 \mathrm{~m}$ \\
\hline Port thickness & $0.012 \mathrm{~m}$ \\
\hline Port angle (lower and upper edge) & $15^{\circ}$ \\
\hline Water model height & $1.20 \mathrm{~m}$ \\
\hline Water model width & $0.50 \mathrm{~m}$ \\
\hline Water model thickness & $0.080 \mathrm{~m}$ \\
\hline
\end{tabular}

\section{Physical Modeling}

A plastic one-third scale water model was designed and constructed in accordance with the Froude similarity criterion. Froude criterion guarantees that the water model is similar to the industrial caster mold from the geometrical and dynamical point of view. ${ }^{8)}$ In this work, the comparison between experimental and numerical results was done by means of a spectral analysis of the numerical simulations. Through the spectral analysis it is possible to distinguish the fundamental structure of possible random signals. With this analysis, some guidelines about operation politics may be stated. The water model, whose geometric dimensions are given in Table 1, is similar to that employed in previous works. ${ }^{4,9,11)}$ The SEN was pool type with two outlet ports with a diameter of $0.02 \mathrm{~m}$, an inlet hole with a diameter of $0.0254 \mathrm{~m}$, height of $0.20 \mathrm{~m}$ and nozzle port angle of $15 \mathrm{deg}$.

It is widely recognized that several process variables have a strong effect on the internal mold flow and therefore, on the slab quality. ${ }^{12-16)}$ Two of the most important process variables are the casting speed and the SEN submergence depth. In water models, variations in the casting speed are obtained by modifying the water volumetric flow rate.

In the continuous casting mold, low values of the inlet molten steel flow rate are a consequence of clogging phenomena. On the opposite, high values of the inlet molten steel flow rate are produced by sudden unclogging phenomena. Besides, high values of the inlet molten steel flow rate are required when the mold level is below its operating point. On the other hand, low values of the inlet molten steel flow rate are required when the free surface level is above its operating point. In this work, three values of the water volumetric flow rate and three values of the SEN submergence depth were considered. Therefore, nine experimental cases arose, which are reported in Table 2. Water volumetric flow rate was varied from $3.50 \times 10^{-4}$ to $5.83 \times 10^{-4} \mathrm{~m}^{3} / \mathrm{s}$, which corresponds to a range of casting speed from 0.9 to $2.5 \mathrm{~m} / \mathrm{min}$. Case 5 corresponds to a typical slab casting mold.

In order to track the transient behavior of the free surface, six measurement positions were defined along the wide wall of the water model, as is shown in Fig. 1. These positions were numerated starting from the mold narrow side wall. The liquid level measurements were made with an ultrasonic distance sensor. The ultrasonic level sensor span is $0.1 \mathrm{~m}$ with $0.05 \mathrm{~m}$ of dead zone and a resolution of $0.00005 \mathrm{~m}$. To ensure identical experimental settings, in each case the SEN submergence depth was measured in non-flow conditions. Thereafter, the pump was turned on. 
Table 2. Experimental Conditions.

\begin{tabular}{|c|c|c|c|}
\hline \multirow{2}{*}{$\begin{array}{c}\text { Experimental } \\
\text { Case }\end{array}$} & \multicolumn{3}{|c|}{ Parameter Values } \\
\cline { 2 - 4 } & $\begin{array}{c}\text { Volumetric flow rate } \\
\left(\mathrm{m}^{3} \mathrm{~s}^{-1}\right)\end{array}$ & $\begin{array}{c}\text { Port output velocity } \\
\left(\mathrm{m} \mathrm{s}^{-1}\right)\end{array}$ & $\begin{array}{c}\text { SEN submergence depth } \\
(\mathrm{m})\end{array}$ \\
\hline 1 & $3.50 \times 10^{-4}$ & 0.557 & 0.05 \\
\hline 2 & $4.67 \times 10^{-4}$ & 0.744 & 0.05 \\
\hline 3 & $5.83 \times 10^{-4}$ & 0.928 & 0.05 \\
\hline 4 & $3.50 \times 10^{-4}$ & 0.557 & 0.07 \\
\hline 5 & $4.67 \times 10^{-4}$ & 0.744 & 0.07 \\
\hline 6 & $5.83 \times 10^{-4}$ & 0.928 & 0.07 \\
\hline 7 & $3.50 \times 10^{-4}$ & 0.557 & 0.09 \\
\hline 8 & $4.67 \times 10^{-4}$ & 0.744 & 0.09 \\
\hline 9 & $5.83 \times 10^{-4}$ & 0.928 & 0.09 \\
\hline
\end{tabular}

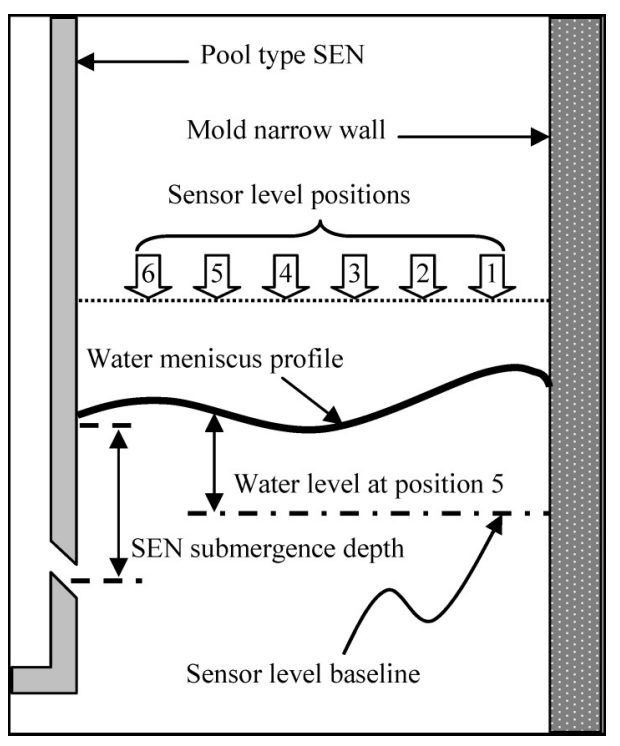

Fig. 1. Schematic representation of the system.

The level measurements were started after the flow was well developed.

The signals of the level sensors were recorded in a computer by means of a data acquisition card at a rate of 10 samples per second during $15 \mathrm{~min}$. This means that 9000 data samples were collected for each sensor. It is important to mention that in some previous works, ${ }^{7,9)}$ only 100 measurements were analyzed.

\section{Results and Discussion}

\subsection{Numerical Results}

Figure 2 shows the free surface profiles for all the experimental cases reported in Table 2. It can be appreciated that the free surface profile strongly depends on the water volumetric flow rate. Assuming that the similarity criterion between the water model and the real steel caster guarantees similar dynamic behavior in both fluids, then one can expect that casting speed affect in the same way the free surface profile of molten steel in the continuous casting mold.

The relationship between SEN output port velocity and free surface profile is consistent with that reported in previous works. ${ }^{4,11)}$ For a constant SEN submergence depth, the wave amplitude is increased as the SEN output port veloci-

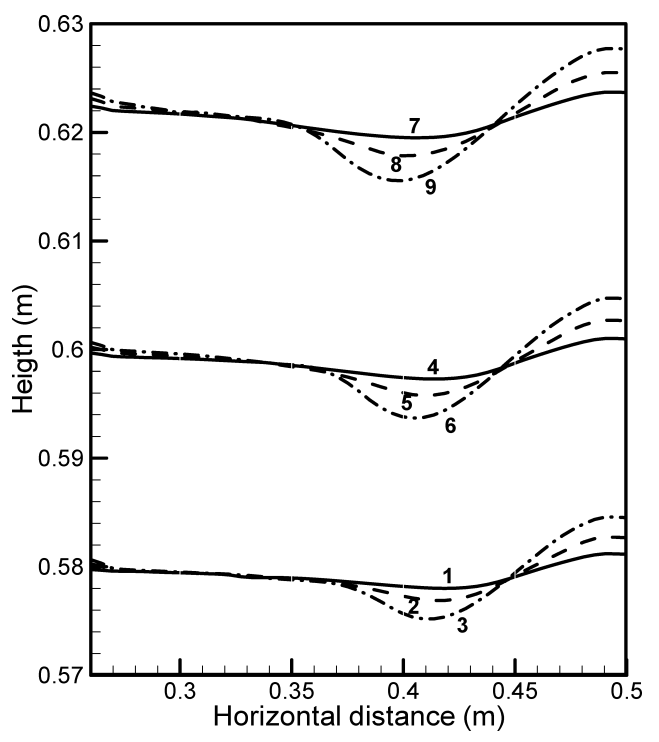

Fig. 2. Free surface profiles for cases 1 to 9 .

ty is increased too. It was observed that in the meniscus zone, the horizontal component of the velocity is predominant and the flow is directed towards the SEN. The water in contact with the narrow wall has a high level due to the ascending flow of the water upper recirculation roll. The lowest water level is present at two-thirds of the distance between the SEN and the narrow wall. Near the SEN, the water level rises and suddenly decreases because the water flow is directed below.

On the other hand, the relationship between free surface profile and SEN submergence depth has been poorly studied. It is interesting to note in Fig. 2 that for a constant SEN output port velocity, the wave amplitude is increased as the SEN submergence depth is increased too. Also, it can be observed that the maximum and the minimum free surface levels moves towards SEN as the SEN submergence depth is increased.

The above characteristics are explained by observing the velocity contours maps in the upper mold section, which are shown in Figs. 3-5. In these figures the cases were ordered in such a way that cases with the same SEN output port velocity are shown in the same figure. It can be observed that the maximum velocity is increased as the SEN submergence depth is increased too. The change in the free 

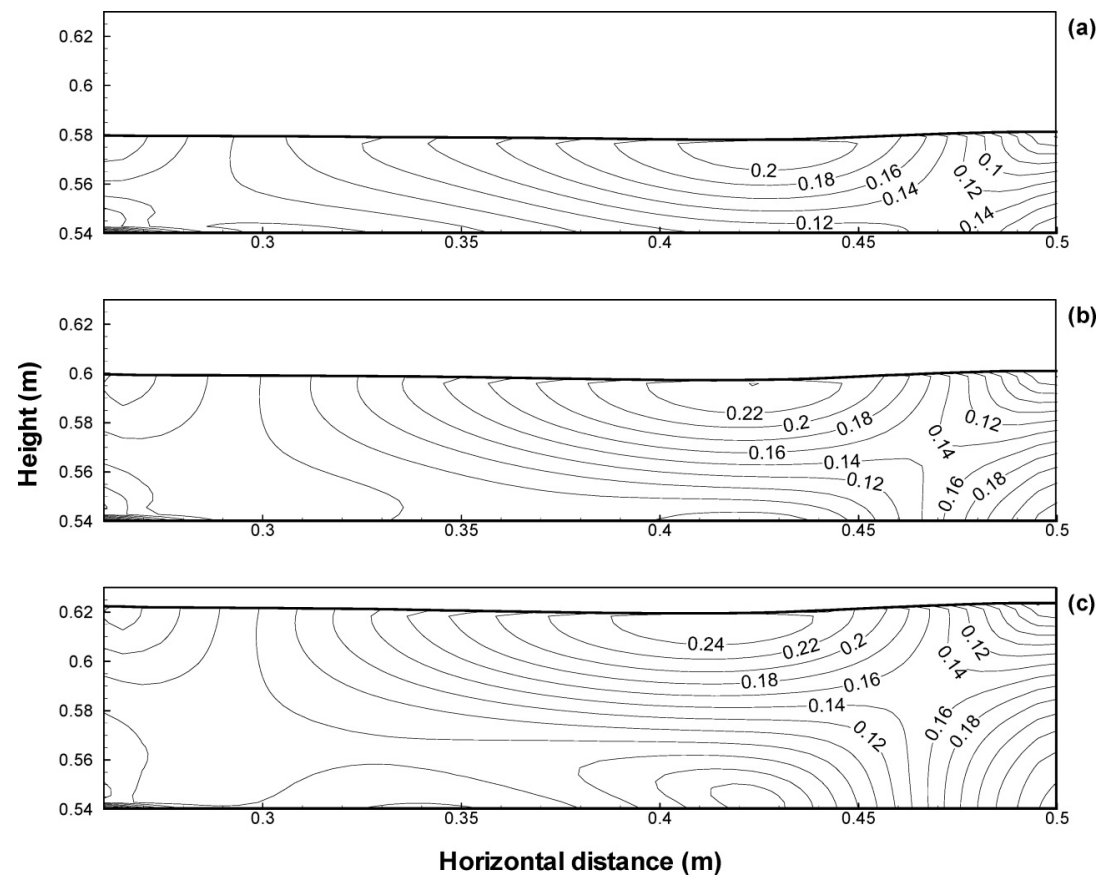

Fig. 3. Contour map of the velocities in the upper mold section. (a) Case 1. (b) Case 4. (c) Case 7.
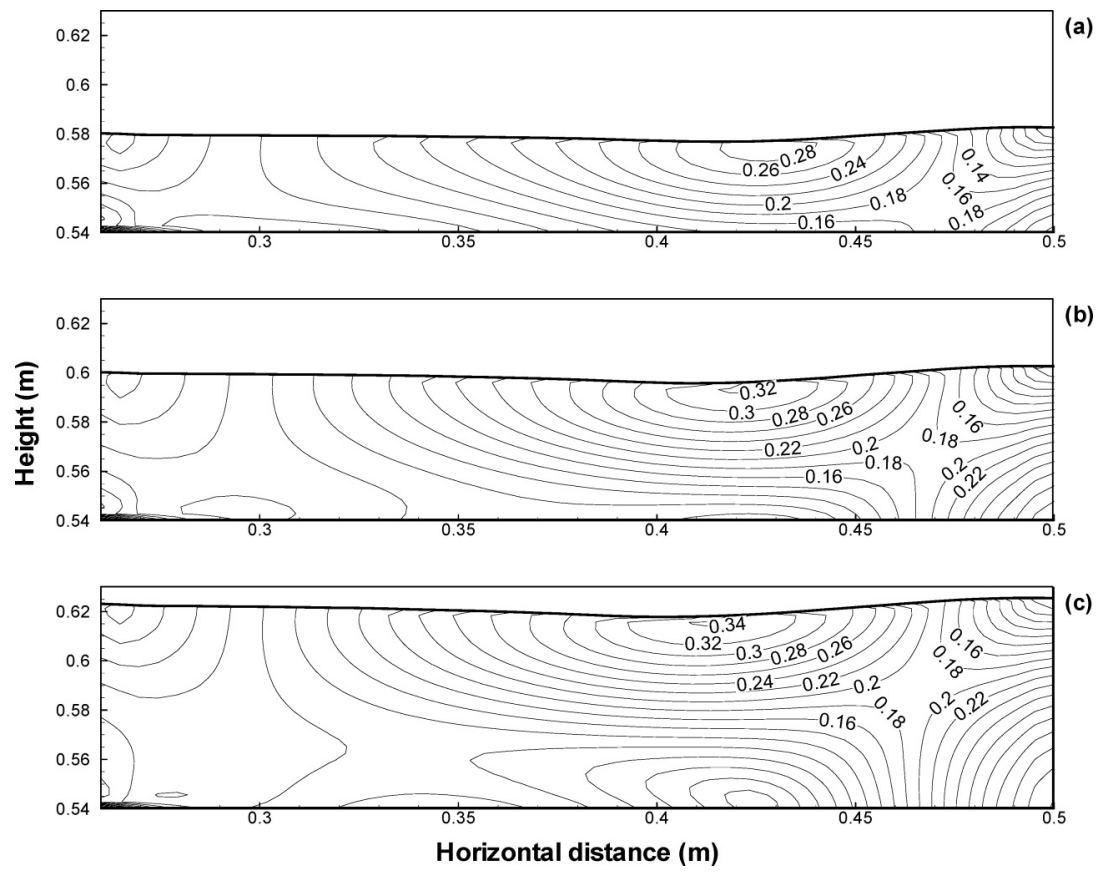

Fig. 4. Contour map of the velocities in the upper mold section. (a) Case 2. (b) Case 5. (c) Case 8 .

surface profile can be explained by observing how the vertical velocity patterns in the upper mold section changes as SEN submergence depth is modified. These patterns are shown in Figs. 6-8.

Roughly speaking, it can be argued that higher velocities in the upper mold section are obtained when the SEN submergence depth is increased because of a great amount of fluid promotes better development of the flow.

\subsection{Physical Results}

Almost all the experimental free surface profiles reported in the literature ${ }^{4,11)}$ were obtained by averaging the measured data over a time period. For data sets measured in this work, the mean and the median are very close. Little indi- cation of skewness was observed in the following data sets: Case 2 at position 1, Case 3 at position 2, Case 4 at position 4, and Case 7 at position 5. Moreover, the histograms and their comparison with normal distributions show that all data sets have not normal distribution. Therefore, in addition to mean or median, data dispersion must be taken into account to characterize the free surface behavior.

\subsubsection{Data Dispersion}

Several authors ${ }^{1,8,12)}$ have claimed that upper recirculation flows are responsible of the free surface oscillations. Additionally, because of their erratic behavior, some authors $^{1,8)}$ have claimed that the free surface fluctuations are chaotic in nature. The free surface level fluctuations ob- 

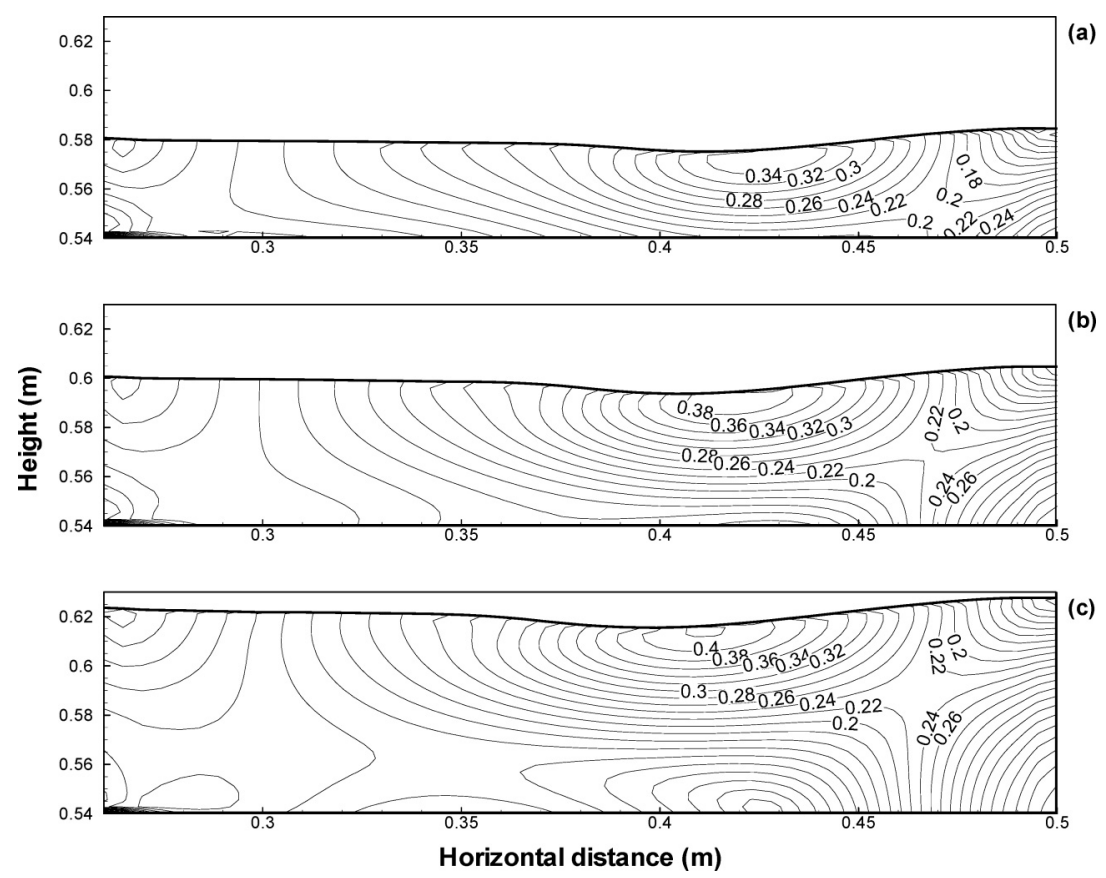

Fig. 5. Contour map of the velocities in the upper mold section. (a) Case 3. (b) Case 6. (c) Case 9.
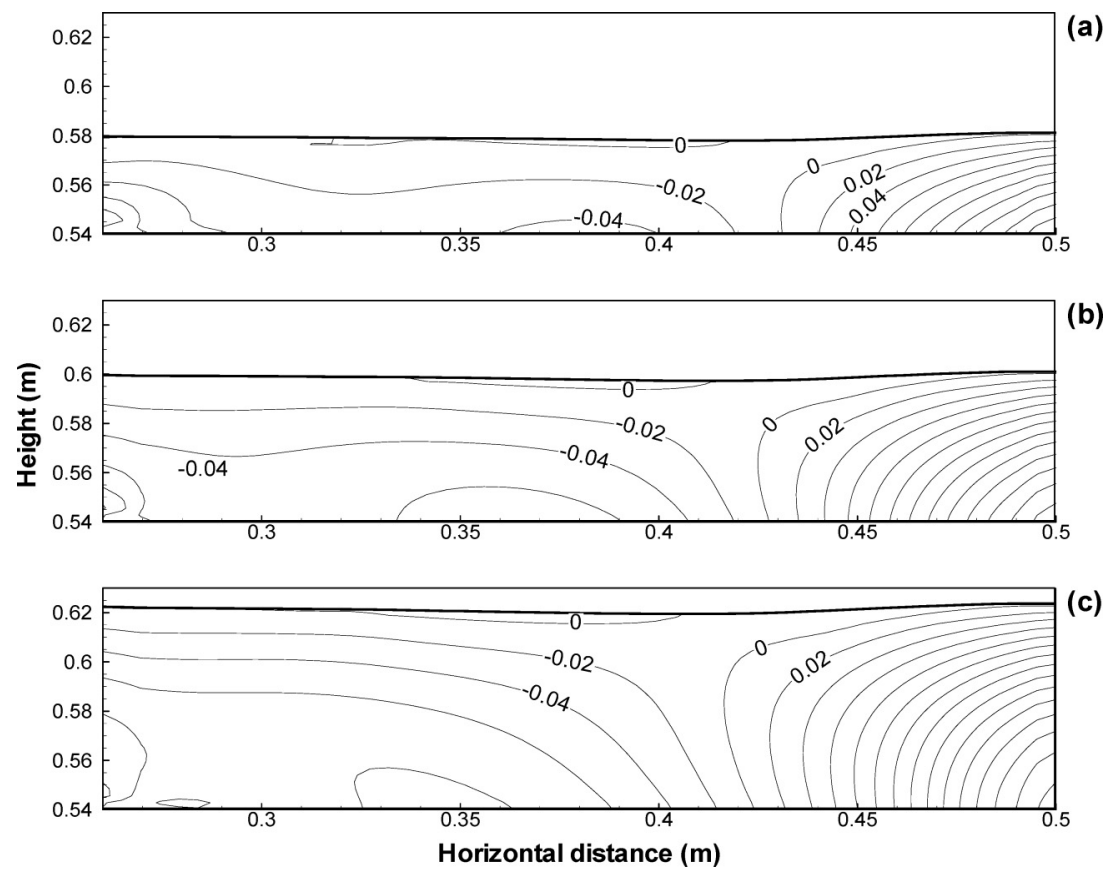

Fig. 6. Contour map of the vertical velocities in the upper mold section. (a) Case 1. (b) Case 4. (c) Case 7.

tained in this work show an erratic behavior. Nevertheless, their nature will be discussed in detail in the next section.

Figure 9 shows the level measurements standard deviation for all the experimental cases. Three groups are formed in accordance with the port output velocity. That is, free surface level standard deviation increases as port exit velocity increases too. Also, the highest values for the standard deviation are obtained at position 2 , because at this position are expected the highest velocity values (see Figs. 3-5).

The dependence of the standard deviation with the SEN submergence depth is appreciated from results of numerical simulations. Given that velocity magnitude increases as SEN submergence depth increases, it could be expected that level measurements standard deviation must increase as SEN submergence depth increase. Nevertheless, from Fig. 9 it is not possible to determine a clear relationship between standard deviation and SEN submergence depth. This result shows that there are phenomena not considered in numerical simulations.

\subsubsection{Free Surface Profile}

A comparison of simulated and experimental free surface for all cases is shown in Figs. 10-12. The filled diamond represents the geometric mean of the experimental free surface level at each measurement position. To capture the largest part of the experimental data, the $3 \sigma$ criterion was 

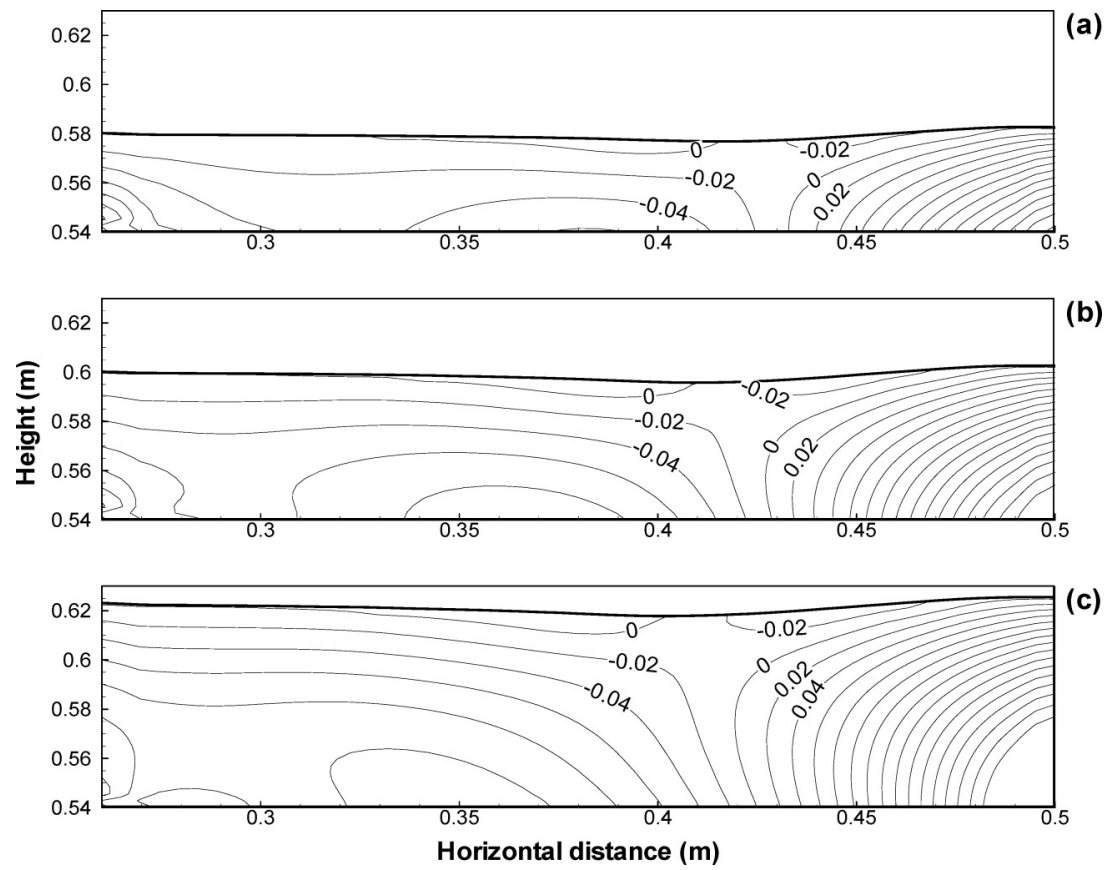

Fig. 7. Contour map of the vertical velocities in the upper mold section. (a) Case 2. (b) Case 5. (c) Case 8.
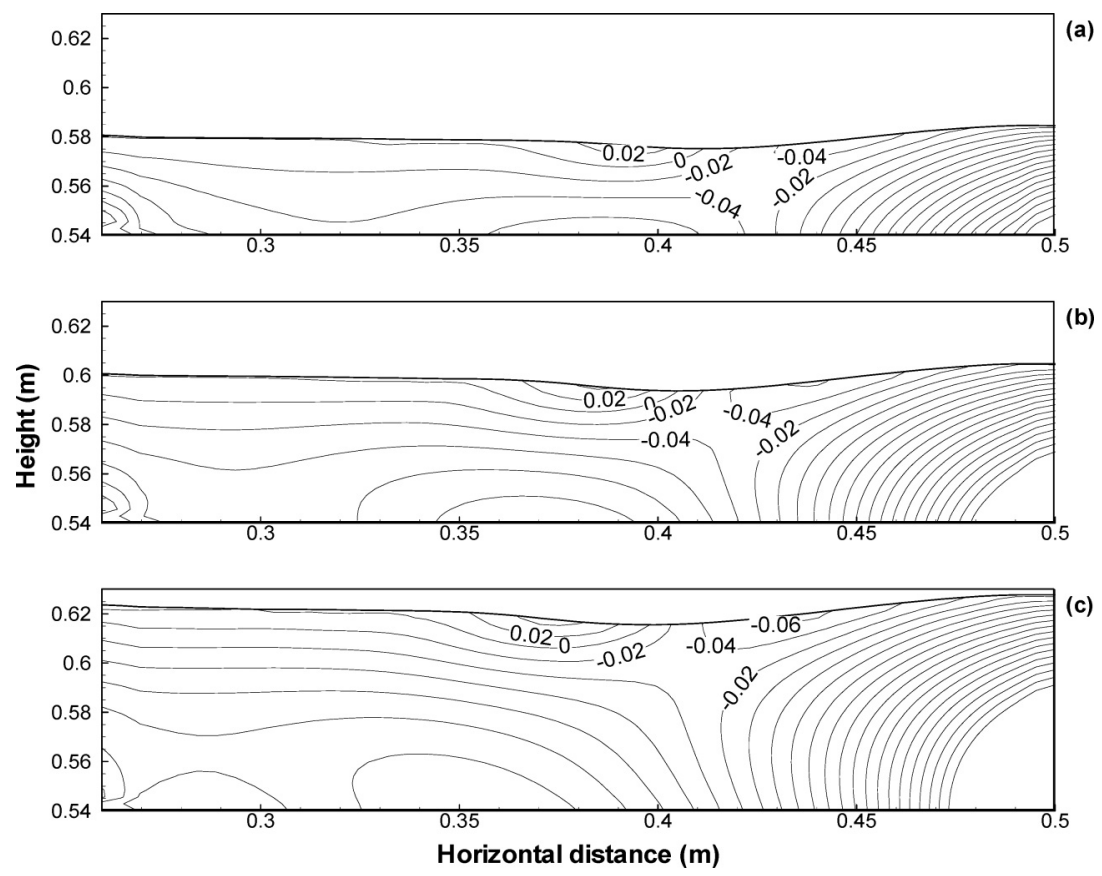

Fig. 8. Contour map of the vertical velocities in the upper mold section. (a) Case 3. (b) Case 6. (c) Case 9.

adopted; therefore, intervals whose size is three times the standard deviation were included. As it can be observed, good agreement between simulated and experimental data was obtained. It can be seen that practically the entire simulated free surface profiles lies inside the experimental interval.

Note that the agreement of the free surface profiles was good enough for Cases 2, 3, 5, 6, 8, and 9. The best agreement between experimental and simulated free surface was obtained for Case 8. The largest differences between numerical and physical simulations were observed at positions 2 and 3, that is, the positions at which the highest velocity magnitudes are expected.

\subsection{Combined Analysis}

Numerical simulations yield the following remarks: (i) When the SEN submergence depth remains constant, the free surface wave amplitude is increased as the SEN output port velocity is increased. (ii) When the SEN output port velocity remains constant, free surface profile is pushed towards the SEN as the SEN submergence depth is increased. (iii) When the SEN output port velocity remains constant, the free surface wave amplitude is increased as the SEN submergence depth is increased too. (iv) When the SEN output port velocity remains constant, the maximum water velocity in upper mold section is increased as the SEN submergence depth is increased. In this work, fluid velocities 


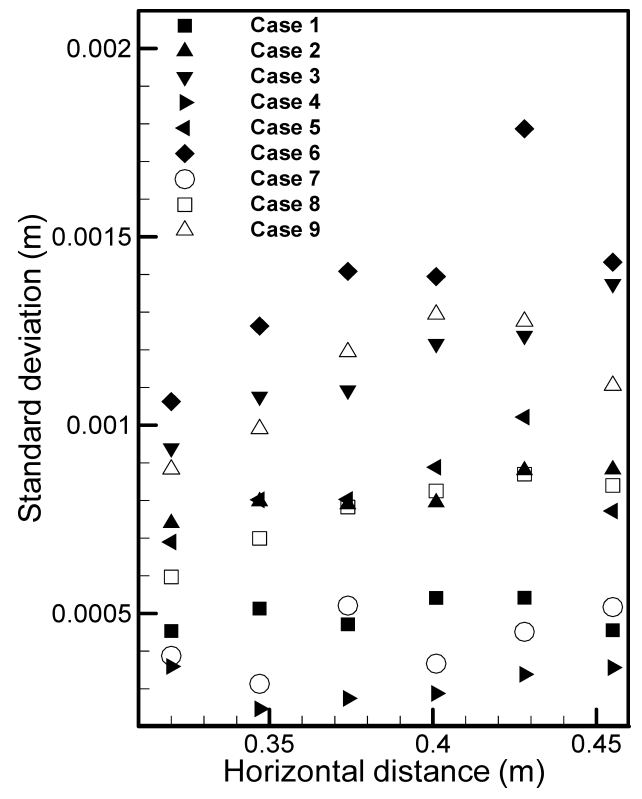

Fig. 9. Experimental standard deviation.

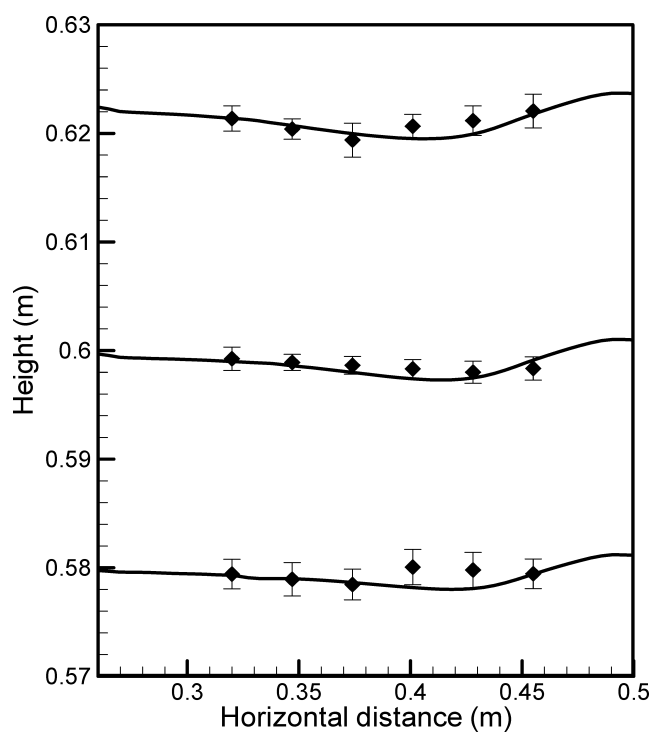

Fig. 10. Comparison of simulated and experimental free surface for Cases 1, 4, and 7. The experimental standard deviation was included.

were not measured, nevertheless remarks (i) and (ii) were corroborated in the physical experiments. On the contrary, remarks (iii) and (iv) are not consistent with the physical experiments.

Some insights about fluid velocities in the upper mold region can be obtained by analyzing the power spectra of the free surface level measurements time series. The power density spectrum (PDS) of a signal represents the contribution of every frequency of the spectrum to the power of the overall signal. The goal of spectral estimation is to describe the distribution on frequency of the power contained in a signal, based on a finite set of data. Given that parametric methods can yield higher resolutions than nonparametric methods in cases when the signal length is short, in this work a parametric method, the Yule-Walker Auto Regressive (AR) method ${ }^{18-20)}$ was employed to calculate the PDS of the measured data. The Yule-Walker method of spectral

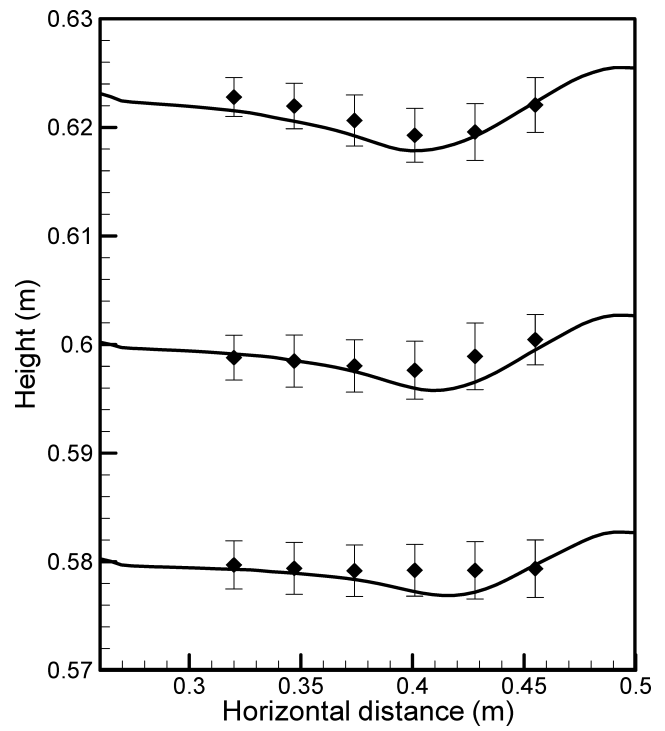

Fig. 11. Comparison of simulated and experimental free surface for Cases 2, 5, and 8. The experimental standard deviation was included.

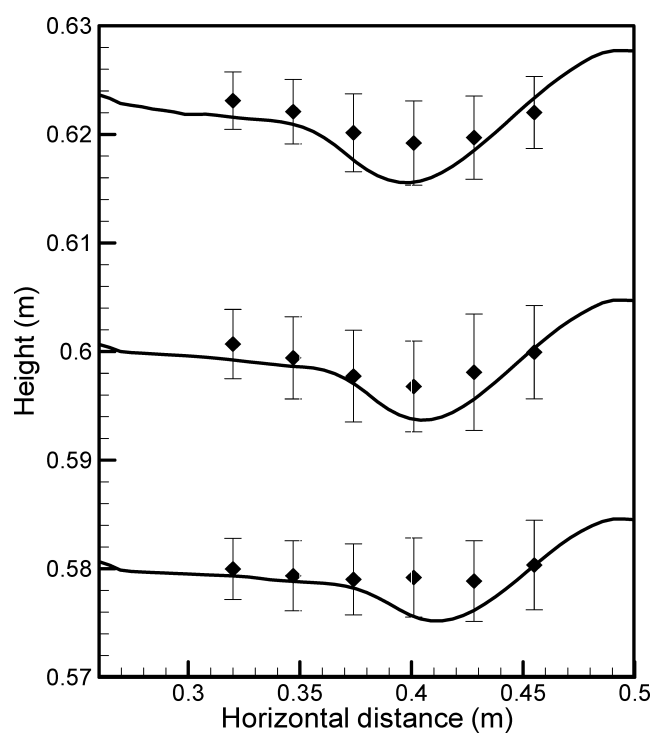

Fig. 12. Comparison of simulated and experimental free surface for Cases 3, 6, and 9. The experimental standard deviation was included.

estimation computes the AR parameters by forming a biased estimate of the signal's autocorrelation function, and solving the least squares minimization of the forward prediction error. The Yule-Walker AR method produces the same results as a maximum entropy estimator. ${ }^{18)}$

Molds similar to the one used in this work have been studied through numerical simulations ${ }^{4,11)}$ using the $K-\varepsilon$ turbulence model. Those authors reported that in the PDS, just one or two peaks were observed. Besides, the frequency of the peaks depends on the port velocity and the spatial position.

Figures 13-18 show the power density spectrum of the experimental data grouped for measurement positions 1-6, respectively. As in infrared spectroscopy, ${ }^{21)}$ the spectrum of each case was multiplied by a constant, which is indicated in the corresponding figure. With this transformation, a better comparison among power spectrums can be done. The 


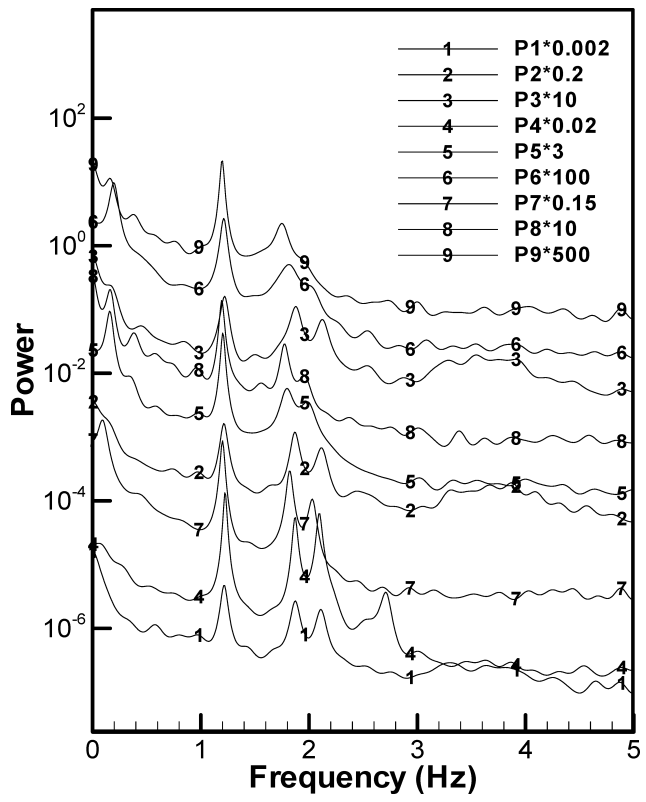

Fig. 13. Power spectrum of the experimental data for all cases at position 1 .

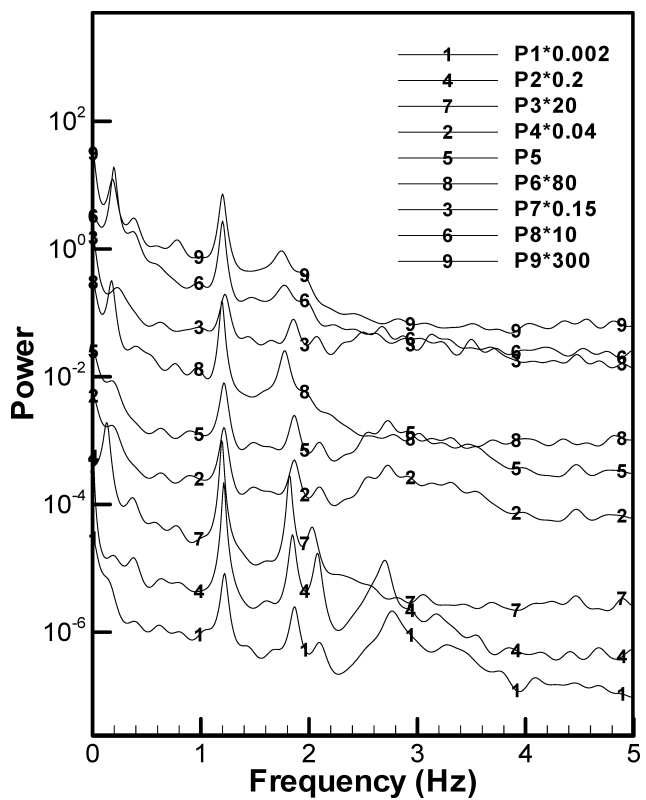

Fig. 14. Experimental data power spectrum for all cases at position 2.

spectrums were grouped in accordance with the port output velocity. In the lower part of the graph are the spectrums with the smaller output port velocity. In the upper part of the graph are the spectrums with the higher output port velocity. Besides, in each group, the spectrum with smaller SEN submergence depth is in the lower part and the spectrum with bigger SEN submergence depth is in the upper part.

From Figs. 13-18 it is clear that there exist multiple periodic components present in all cases at all positions. However, there is one periodic component present in all cases at all positions whose frequency is around $1.2 \mathrm{~Hz}$. This periodic component will be used as a reference point in the subsequent analysis. The present authors believe that this frequency is related with the SEN design. There are an-

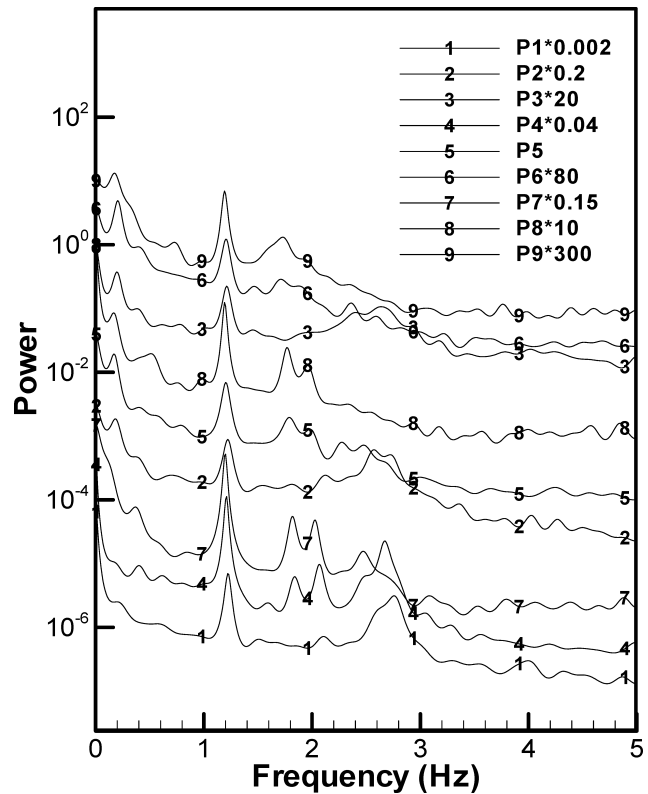

Fig. 15. Power spectrum of the experimental data for all cases at position 3 .

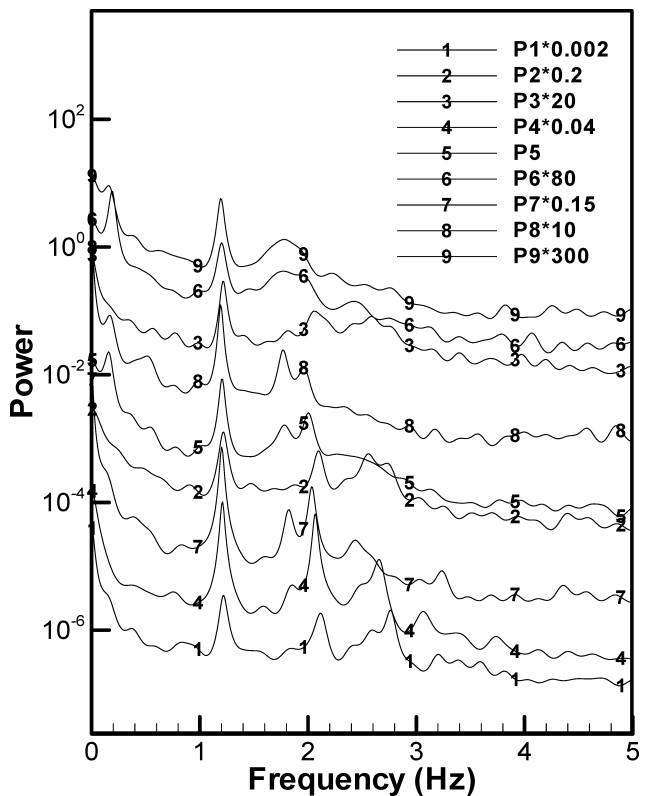

Fig. 16. Power spectrum of the experimental data for all cases at position 4 .

other two main periodic components present, with frequencies around $1.8 \mathrm{~Hz}$ and $2.1 \mathrm{~Hz}$. These values are close to that reported in previous works. ${ }^{4}$ For a constant SEN submergence depth, these two periodic components become less significant as the port output velocity is increased.

It is interesting to note that for a constant port output velocity, the following behavior was observed: (i) Periodic components with characteristic frequency higher than $1.2 \mathrm{~Hz}$ become less significant as the SEN submergence depth is increased, (ii) Periodic components with characteristic frequency smaller than $1.2 \mathrm{~Hz}$ become more significant as the SEN submergence depth is increased.

By combining the experimental results with that obtained through numerical simulations, the following assertions can be stated: (i) Periodic components with characteristic fre- 


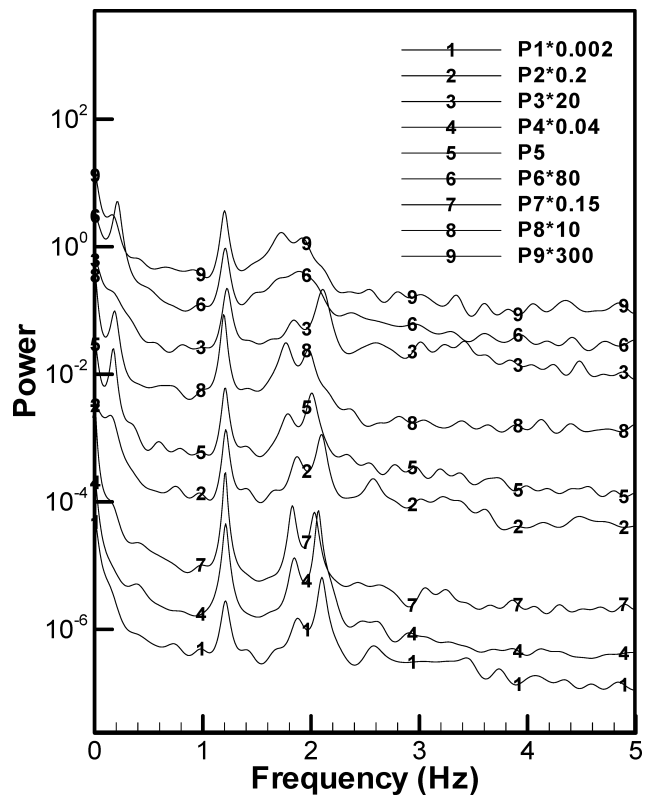

Fig. 17. Power spectrum of the experimental data for all cases at position 5 .

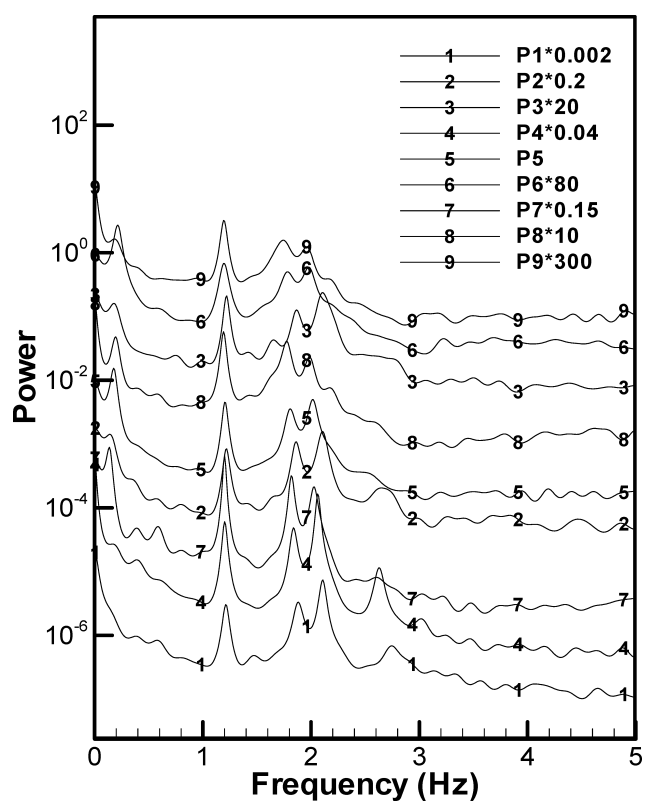

Fig. 18. Power spectrum of the experimental data for all cases at position 6 .

quency higher than the mold fundamental frequency of $1.2 \mathrm{~Hz}$ reflect erratic behavior of the fluid in the mold upper zone, and (ii) Periodic components with characteristic frequency lower than the mold fundamental frequency reflect an organized behavior of the fluid in the same mold zone. So, it can be expected that higher fluid velocities can be obtained in cases in which the low frequency periodic components are more significant than high frequency periodic components. When analyzing horizontal velocities below top surface, Thomas et al ${ }^{8)}$ arrived to similar conclusions using large eddy simulations and particle image velocimetry measurements. They pointed out that low frequency components are of significant interest as they would likely exacerbate shear entrainment of the liquid flux at the top surface and level fluctuations.
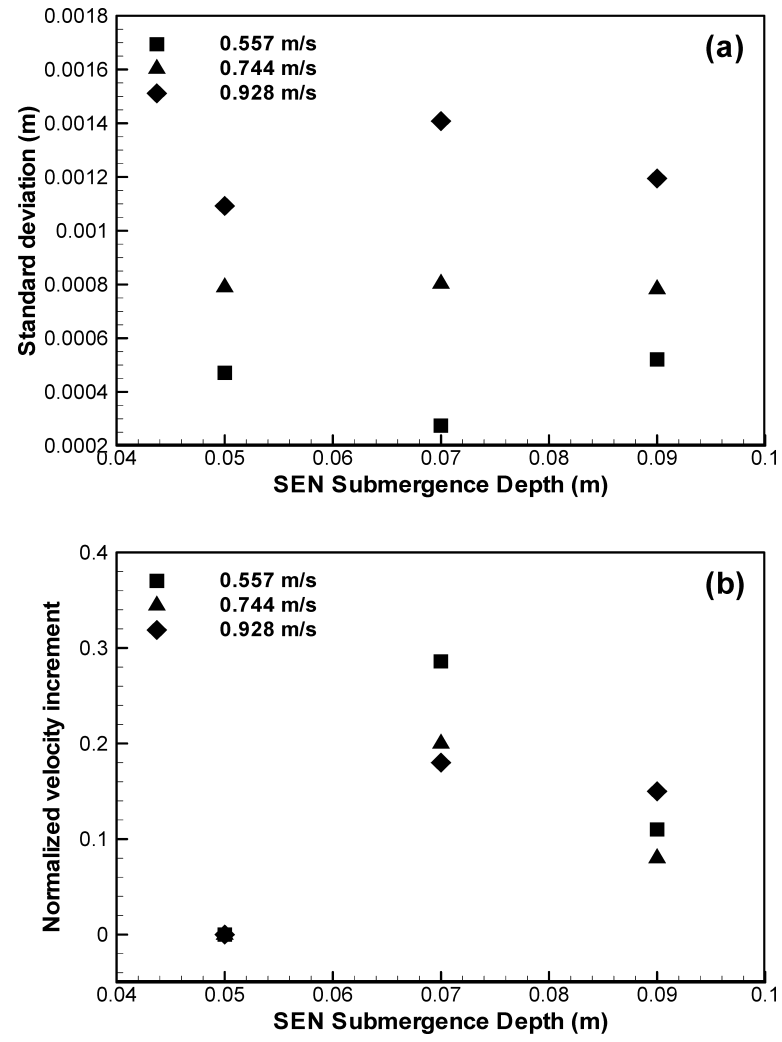

Fig. 19. (a) Standard deviation and (b) normalized velocity increment for all cases at number 4 sensor position.

It is interesting to analyze the influence of the SEN submergence depth on the surface velocity. Figure 19(a) shows the experimental standard deviation for all cases considered at the number 4 sensor position. Figure 19(b) shows the normalized velocity increment, at the same sensor position, which was calculated as the ratio of the surface velocity difference between the considered and the previous SEN submergence depths and the considered one. In accordance with Figs. 19(a) and 19(b), for small SEN submergence depths the surface velocity increases as submergence depth increases too, as reported by Lawson and Davidson. ${ }^{22)}$ However, for a certain value of the SEN submergence depth the opposite behavior is observed, that is, the surface velocity decreases as submergence depth is increased. In this work, the above value of the SEN submergence depth is named as Critical SEN Submergence Depth. The free surface profile corresponding to this critical SEN submergence depth is named as Critical Free Surface Profile. For low port exit velocities the aforementioned behavior is not observed probably due to in these situations the high frequency components of the surface velocity are significant, therefore, the dominant behavior is mainly erratic.

Considering that the analysis and the results presented in this work are applicable to industrial slab continuous casting machines, several suggestions regarding the operation can be stated: (i) Given that for a constant casting speed there is a maximum in the relation between velocity in the upper mold section and SEN submergence depth, the free surface profile at which this maximum takes place must be determined. With this information the best control politics can be determined. (ii) For a constant port output velocity, the probability of slag trapping is increased in situations in 
which the current free surface profile tends to decrease and is above the critical free surface profile. This can occur under nozzle clogging situations or when casting speed is suddenly increased. (iii) Besides, the probability of slag trapping is increased in situations in which the current free surface profile tends to increase and is below the critical free surface profile. This can occur under nozzle unclogging situations or when casting speed is suddenly decreased.

\section{Concluding Remarks}

The power density spectrum of the free-surface of a slab mold water model is composed by several periodic components, the most important being those of 1.2, 1.8 and $2.1 \mathrm{~Hz}$. The dependence of these components on the port output velocity and SEN submergence depth was studied. For SEN submergence depths below a critical value, the contribution of high frequencies to the free surface dynamic behavior decreases as SEN submergence depth is increased. For SEN submergence depths above that critical value, an opposite behavior is observed. Determining the way in which the periodic components of the free surface profile can be modified, could improve eventually the quality of the steel produced.

\section{Acknowledgments}

Raul Miranda (R. M.) thanks to Consejo Nacional de Ciencia y Tecnología (CONACYT) for Grant No. 164887. This work was done in partial fulfillment of R.M.'s PhD requirements.

\section{REFERENCES}

1) S. Kumar, B Walker, I. Samarasekera and J. Brimacombe: PTD Conf. Proc., Warrendale, PA, (1995), 119.
2) M. Dussud, S. Galichet and L. Foulloy: IEEE Trans. Control Syst. Tech., 6 (1998), 246.

3) M. Iguchi, K. Hanazaki and D. Iguchi: ISIJ Int., 43 (2003), 836.

4) G. A. Panaras, A. Theodorakakos and G. Bergeles: Metall. Mater. Trans. B, 29B (1998), 1117.

5) M. Barrón, R. Aguilar and J. González: IEE Proc. Cont. Theory Appl., 147 (2000), 416

6) T. Watanabe, K. Omura, K. Furukawa, S. Watanabe and M. Konishi: Trans. Soc. Inst. Cont. Eng., 36 (2000), 884.

7) D. Gupta, S. Chakraborty and A. Lahiri: Metall. Mater. Trans. B, 25B (1994), 227.

8) B. G. Thomas, Q. Yuan, S. Sivaramakrishnan, T. Shi, S. P. Vanka and M. B. Assar: ISIJ Int., 41 (2001), 1262.

9) D. Gupta, S. Chakraborty and A. Lahiri: ISIJ Int., 37 (1997), 654.

10) K. Takatani, Y. Tanizawa, H. Mizukami and K. Nishimura: ISIJ Int., 41 (2001), 1252.

11) A. Theodorakakos and G. Bergeles: Metall. Mater. Trans. B, 29B (1998), 1321.

12) B. G. Thomas, Q. Yuan, S. Sivaramakrishnan and S. Vanka: JOM-e (Journal of Metals - electronic edition) (2002).

13) Y.-H. Ho, C.-H. Chen and W.-S. Hwang: Ironmaking Steelmaking, 28 (2001), 258.

14) U.-S. Yoon, W. Bang, J. H. Rhee, S.-Y. Kim, J.-D. Lee and K. H. Oh: ISIJ Int., 42 (2002), 1103.

15) E. Hoffken, H. Laz and G. Pietzko: Proc. of the 4th Int. Conf. Continuous Casting, Brussels, (1988), 461.

16) N. Kubo, J. Kubota and T. Ishii: ISIJ Int., 41 (2001), 1221.

17) P. Z. Peebles, Jr.: Probability, Random Variables, and Random Signal Principles, 3rd Ed., McGraw-Hill, New-York, (1993), 199.

18) J. G. Proakis and D. G. Manolakis: Digital Signal Processing: Principles, Algorithms, and Applications, Prentice Hall, New Jersey, (1996), 850.

19) D. B. Percival and A. T. Walden: Spectral Analysis for Physical Applications: Multitaper and Conventional Univariate Techniques, Cambridge University Press, Cambridge, (1993), 510.

20) R. M. Howard: Principles of Random Signal Analysis and Low Noise Design: The Power Spectral Density and its Applications, Wiley-Interscience, New York, (2002), 114.

21) J. W. Niemantsverdriet: Spectroscopy in Catalysis. An Introduction, 2nd Ed., Wiley-VCH, Netherlands, (2000), 167.

22) N. J. Lawson and M. R. Davidson: J. Fluids Eng., 124 (2002), 535. 